\title{
Duvelisib Regimen
}

National Cancer Institute

\section{Source}

National Cancer Institute. Duvelisib Regimen. NCI Thesaurus. Code C160027.

A chemotherapy regimen consisting of duvelisib that may be used in the treatment of chronic lymphocytic leukemia (CLL), small lymphocytic lymphoma (SLL), and follicular lymphoma. 